\title{
Metodología de planificación estratégica para instituciones de salud a partir de valores compartidos
}

\section{Strategic planning methodology for health institutions on the basis of shared values}

\author{
Yuslay Ponce Sánchez'; Alfredo Pardo Fernández"'; Carmen Arocha \\ Mariño"'; J uan Carlos Rojas Fernández ${ }^{\text {IV }}$ \\ 'Máster en Salud Pública. Instructora. Centro Provincial de la Escuela Nacional de \\ Salud Pública. Guantánamo, Cuba. \\ "Doctor en Ciencias Pedagógicas. Profesor Titular. Centro Provincial de la Escuela \\ Nacional de Salud Pública. Guantánamo, Cuba. \\ IIIDoctora en Ciencias de la Salud. Profesora Titular. Escuela Nacional de Salud \\ Pública. La Habana, Cuba. \\ IVEspecialista de I Grado en Medicina General Integral. Instructor. Centro Provincial \\ de la Escuela Nacional de Salud Pública. Guantánamo, Cuba.
}

\section{RESUMEN}

La sociedad cubana trabaja en el fortalecimiento de la educación en valores lo que implica cambios organizacionales que dependen del cambio del comportamiento de los miembros de la organización y este de sus valores. Para contribuir al logro de lo planteado, se elaboró una metodología para la realización de planes estratégicos a partir de los valores compartidos por los trabajadores de las instituciones de salud. Se obtuvo información sobre metodologías existentes para la elaboración de planes estratégicos generales. Dentro de las encontradas se seleccionó una como patrón por considerarse la más adecuada. Se diseñaron y aplicaron instrumentos para el diagnóstico de los valores actuales y técnicas para la determinación de los valores deseados. La metodología se estructuró en tres etapas: la primera se denominó Análisis Situacional, que se corresponde con el estado presente de la institución, aquí se evalúan los valores actuales compartidos. En la segunda etapa, denominada Prospectiva, se valora el futuro institucional y por tanto tiene en cuenta la determinación de los valores deseados, la tercera etapa se denominó Operacional o de conexión del presente con el futuro, en ella se elaboran los valores instrumentales. Mediante la aplicación de esta metodología los directivos planifican 
el cambio organizacional a partir de la intención de cambiar el comportamiento moral de los miembros de la organización. Ha sido validada por criterio de expertos y por su empleo en la conformación de 141 planes estratégicos de departamentos, servicios e instituciones de salud de la provincia de Guantánamo, con buenos resultados.

Palabras clave: Metodologías, planificación estratégica, valores, valores compartidos.

\begin{abstract}
The Cuban society is working in the strengthening of ethical values education, which leads to organizational changes depending on the change of the organization members's behaviors and values. For achieving the above-mentioned, a methodology was drawn up to carry out strategic plans based on the values that the health institution workers share. Data on already existing methodologies to work out general strategic plans were collected. One methodology was selected as pattern because it was considered the most appropriate. Some diagnostic instruments to identify current values and some techniques to ascertain desired values were designed and applied. The methodology structure involves three phases: the first phase called Situation Analysis covers the present state of the institution and evaluates the current shared values. The second phase called Prospective assesses the institutional future and hence determines the desired values whereas the third phase called Operational or Linking, which relates the present and the future, works out the instrumental values. Through the implementation of this methodology, the managers are able to plan the organizational change on the basis of the intention of changing the moral behavior of the members. This methodology has been validated by expert criteria and use in the drafting of 141 strategic plans at department, service and health institution levels in Guantanamo province, with good results.
\end{abstract}

Key words: Methodologies, strategic planning, values, shared values.

\title{
I NTRODUCCI ÓN
}

A principios del siglo xx, fue conceptualizada teóricamente la Dirección por Instrucciones (Dpl) [García S, Simón D. La Dirección por Valores. La Habana; 1999]. Representaba la época en la que se podía lograr el éxito manejando los recursos organizativos externos como el control, la disciplina y el cumplimiento de la jornada, entre otros. Tradicionalmente la Dpl se manifiesta en situaciones repetitivas de baja complejidad, donde es necesario dar respuestas simples y automáticas.

La Dpl basada en jerarquías y funciones dio lugar a la Dirección por Objetivos (DpO) a partir de la década de los 60s, como un movimiento que va de la disciplina férrea al compromiso consciente con las tareas para lograr el éxito, constituye un 
sistema administrativo, dirigido hacia el logro eficaz y eficiente de objetivos organizacionales e individuales. ${ }^{1}$

En la práctica sucede que a veces la DpO deja de ser todo lo eficiente y motivadora que debería ser y se rechaza porque no se aplica de forma suficientemente participativa. Se necesita entonces transitar de la $\mathrm{DpO}$ a una dirección participativa por objetivos (DPpO). Al ofrecer participación plena a los miembros de una organización en los procesos crece la complejidad organizativa, y no es una tarea fácil reducir estos niveles de conflictos.

En las últimas décadas (alrededor de los años 1990) y como consecuencia del aumento de la complejidad organizativa, aparece la Dirección por Valores (DpV) como un nuevo estilo de gestión, basado en los valores como inspiradores e impulsores de fundamentales y trascendentales cambios. La DpV desarrolla una nueva forma de influir positivamente en la vida de los individuos haciendo más humana la actividad de dirección. [García S, Simón D. La Dirección por Valores. La Habana; 1999].

Para llevar a cabo los procesos de DpO y DpV, la Planeación Estratégica es imprescindible, sobre todo cuando los objetivos o fines existenciales de la organización necesitan ser trazados a largo, mediano y corto plazo y ser conocidos, asimilados y compartidos entre los miembros de la organización, como valores. Es necesaria entonces, la participación de los trabajadores en la determinación de estos objetivos y en el resumen final de los valores ético-morales compartidos entre los miembros de la organización como elementos de la cultura que determinan el comportamiento organizacional.

En Cuba, desde el triunfo revolucionario se humanizó la actividad del hombre y promovió un liderazgo acorde con el sentido humanista del proceso revolucionario. El Comandante Ernesto Ché Guevara, en carta a Carlos Quijano del periódico "Marcha", especifica el método de dirección usado por Fidel en el Ejército Rebelde en la Sierra Maestra. El Che escribe:

...De allí que sea tan importante elegir correctamente el instrumento de movilización de las masas. Este instrumento debe ser de índole moral, fundamentalmente, sin olvidar una correcta utilización del estímulo material, sobre todo de naturaleza social...

..A la cabeza de la inmensa columna - no nos avergüenza ni nos intimida decirlo- va Fidel, después, los mejores cuadros del Partido, e inmediatamente, tan cerca que se siente su enorme fuerza, va el pueblo en su conjunto; sólida armazón de individualidades que caminan hacia un fin común; individuos que han alcanzado la conciencia de lo que es necesario hacer $^{2} .$.

El 19 de abril de 1986, Fidel expresó: "Creo que los problemas hay que resolverlos también con moral, con honor, con principios y es necesario apelar -y sería demagógico no hacerlo- al sentido del deber de nuestros compatriotas y de nuestros trabajadores. " ${ }^{3}$ Incita el máximo líder a desarrollar un nuevo estilo para la solución de los problemas invocando al sentido del deber, a la ética organizacional, a los valores como única vía para implementar una dirección democrática y más humana.

Recientemente una comisión designada por el Comité Central del Partido Comunista de Cuba ha elaborado el Programa Director para el reforzamiento de valores fundamentales en la sociedad cubana actual. ${ }^{4}$ Este documento rector parte de la 
necesidad de profundizar y fortalecer la educación en valores en todas las entidades socializadoras de la sociedad cubana.

El Ministro de Salud Pública J osé Ramón Balaguer Cabrera, en el taller nacional de preparación metodológica de profesores de la Escuela Nacional de Salud Pública realizado en 1994, materializa conceptualmente estas ideas al exponer la necesidad del desarrollo de una actitud nueva ante el trabajo en salud, a través del concepto de Colectivo Moral, en el cual se han de fundir los elementos de la excelencia de la alta tecnología, con la excelencia de la condición humana del trabajador y expresa que:

...La idea del colectivo moral surgió cuando se analizaba la conducta y los valores de los trabajadores de la Salud Pública, donde la moral, la ética, la entrega total, el amor al ser humano deben caracterizar al trabajador de la salud, es por ello, que no se puede alcanzar la excelencia basado en la alta tecnología, es necesaria la concepción del colectivo moral, es necesario considerar la preparación ética, política e ideológica del cuadro de manera que dentro de su responsabilidad esté de forma priorizada el carácter formador para con los trabajadores que dirige...

Estas ideas se concretan en el trabajo teórico-práctico del Centro Provincial de la Escuela Nacional de Salud Pública (CPENSAP) de Guantánamo que tiene como objeto capacitar a los cuadros, reservas y canteras del sector a través de los diplomados de dirección I y II, cuyos contenidos están en correspondencia con lo expuesto por el ministro Balaguer.

A partir de estos diplomados se han elaborado los proyectos de transformación grupal de 64 unidades de salud y 1452 proyectos de transformación individual (CPENSAP de Guantánamo. Informe oficial a la dirección nacional de cuadros del MINSAP. Junio de 2008).

Estos informes han contribuido a conformar un banco de problemas entre los cuales está la intención de los directivos de implementar la dirección por valores. ${ }^{5}$

Esta dirección se limita a listar un grupo de valores y antivalores sin hacer una evaluación organizacional que les permita acercarse a los valores o antivalores compartidos; todavía no se comprende que el cambio organizacional que se proponen depende del cambio de comportamiento de los miembros de la organización y este de sus valores. Las estrategias existentes declaran valores actuales y deseados, pero no existen acciones dirigidas a operacionalizar su alcance. El Análisis de la Situación de Salud no es un instrumento importante para el trazado estratégico y la toma de decisiones.

Teniendo en cuenta las insuficiencias enunciadas anteriormente, es indudable la necesidad de que se elaboren los planes estratégicos en las instituciones de salud a partir de la integración de la dirección por objetivos y la dirección por valores, de ahí que el objetivo de este trabajo es elaborar una metodología para la realización de planes estratégicos a partir de los valores compartidos por los trabajadores en las instituciones de salud.

\section{MÉTODOS}


El método utilizado consistió en la revisión bibliográfica sobre diferentes metodologías para la elaboración de planes estratégicos. Entre las consultadas se decidió utilizar como patrón el modelo aportado por el Doctor en Ciencias Pedagógicas Alfredo Pardo Fernández $z^{6}$ en su tesis doctoral y realizar los ajustes necesarios para insertar los valores en las diferentes etapas que contiene la metodología que se propone. El citado modelo (figura 1) estructura una contradicción dialéctica entre el estado actual y el estado deseado, los cuales se excluyen y complementan mutuamente, de lo que resultan planes estratégicos que se componen de tres etapas.

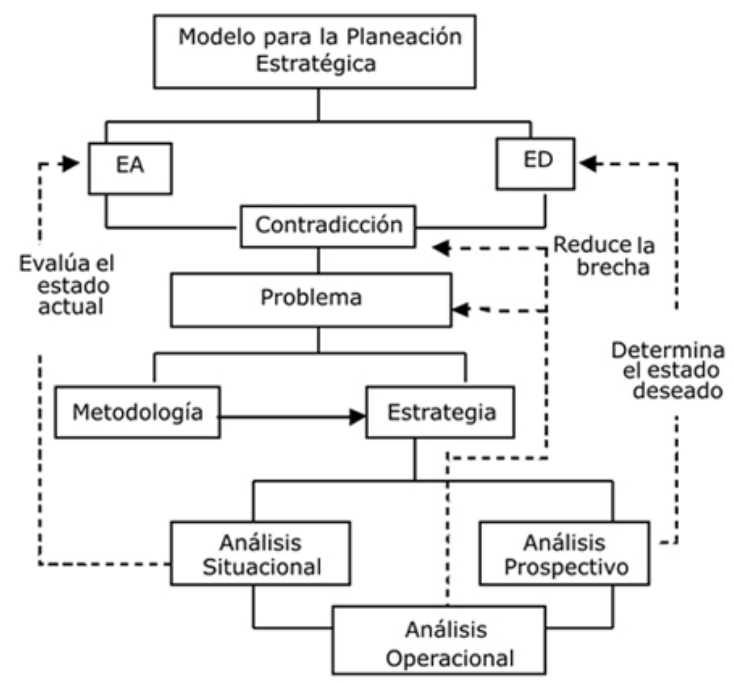

Fig. 1.

En la metodología que se propone se tuvieron en cuenta el sistema de valores compartidos por los trabajadores de la organización. Y se conceptualizaron como valores actuales: aquellos que están presentes en la organización, son objetivos y los comparten la mayoría de los trabajadores, como valores deseados aquellos que no están presentes en la organización y son aspiraciones que a veces se encuentran en el sistema oficial de valores y los valores instrumentales como los que se expresan como metas consensuadas mediante la combinación dinámica de los valores actuales con los deseados, constituyen el elemento dinámico para el cambio pues se convierten en normas de conductas que regulan, desde la subjetividad y la acción valorativa grupal el comportamiento de los trabajadores.

Posteriormente se hizo un análisis del modelo de partida, consideraciones teóricas de las tres etapas de la planeación estratégica, se elaboró un glosario con el significado de los valores que se evalúan, se diseñaron herramientas para la evaluación de los valores actuales, se conformó la matriz de ranqueo para determinar el orden de jerarquización de los valores, se propusieron instrumentos para hacer más pertinente la planeación y poder trazar acciones encaminadas a intervenir en los problemas de salud de la población. Para todos los pasos se organizaron grupos focales y se tuvo en cuenta el criterio de expertos.

\section{Metodología para la realización de planes estratégicos a partir de los valores compartidos por los trabajadores de las instituciones de salud}

La representación esquemática de la metodología que se propone aparece reflejada en la figura 2 , donde se puede observar que en el análisis situacional se realiza la evaluación de los valores actuales; en el análisis prospectivo se determinan los 
valores deseados y en el análisis operacional se elaboran los valores instrumentales.

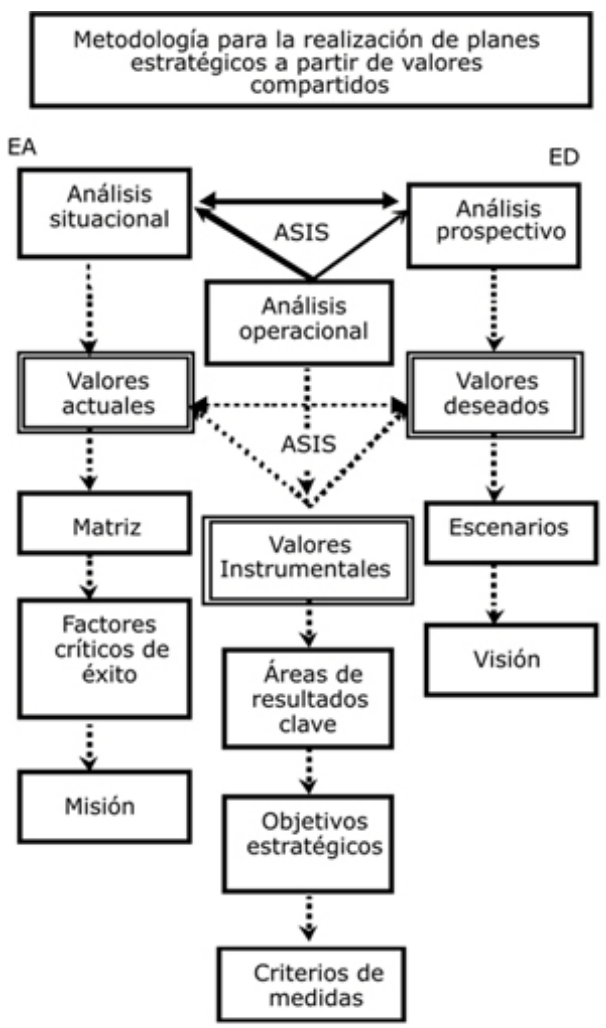

Fig. 2.

En la primera etapa (Análisis Situacional) es necesario hacer una evaluación de los valores compartidos actuales, para esto, se elabora un glosario que se discute y aprueba con los trabajadores que participan en el estudio. Luego se aplica un cuestionario que permite hacer una evaluación cuantitativa y cualitativa de los valores compartidos, donde se obtienen valores y antivalores éticos. ${ }^{7}$

Los resultados de este instrumento se llevan a los grupos focales, donde los trabajadores aprueban o no el resultado del cuestionario y construyen la significación compartida de cada valor y de cada antivalor.

Para completar la evaluación de los valores compartidos, es necesario completar la triangulación metodológica consultando el criterio de los expertos. Estos realizan el ejercicio de evaluar a través de una matriz de ranqueo los valores y antivalores obtenidos, partiendo de la correspondencia de estos con tres variables: ${ }^{8}$

1). La objetividad que expresa la medida en que el valor se manifiesta en el comportamiento de las personas. 2). La socialización, medida en que el valor es compartido por un mayor número de personas en la organización. 3). La dinámica o forma en la que el valor es útil para la solución de los problemas de la institución. Esta se calcula de la misma manera que la matriz tradicional de ranqueo de problemas.

\begin{tabular}{|c|c|c|c|}
\hline Valores & Objetividad & Socialización & Dinámica \\
\hline
\end{tabular}




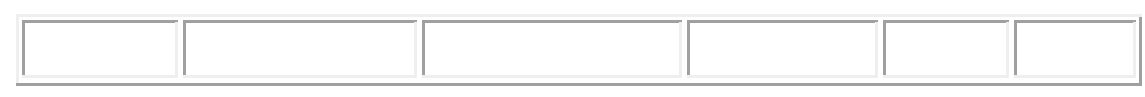

Una vez que se obtiene la lista final de los valores y antivalores en orden de jerarquización o importancia, entonces se determinan los problemas de la organización. Estos se establecen utilizando una guía de observación para examinar los Análisis de la Situación de Salud y obtener los que tienen relación con el estado de salud de la población. A partir del modelo propuesto por Hubert Laframboise y Marc Lalonde, se tienen en cuenta los estilos de vida, el medio ambiente, la organización de los servicios de salud y la biología humana. ${ }^{9}$

Mediante un árbol de problemas se generalizan los problemas específicos a problemas intermedios y de estos a un problema global, luego se procede a determinar cuales de los valores obtenidos alcanzaron significación en relación con los problemas. A esto se llega, a través de la siguiente pregunta: ¿Qué antivalores éticos y profesionales están ocasionando este problema?, de la misma forma, se determinan los valores que alcanzaron una significación positiva para la solución de los problemas. ¿Qué valores éticos y profesionales permiten solucionar este problema? Así se contribuye a determinar el alcance objetivo del valor como regulador del comportamiento deseable o indeseable por el colectivo.

Para determinar la capacidad que poseen estos valores para defender o hacer vulnerable a la institución, se contrastan con las oportunidades y amenazas del entorno. La contrastación entre los factores internos y los externos se realiza a través de la Matriz de Balance de Fuerzas Innovada (BAFI) ${ }^{10}$ Esta matriz evalúa el estado actual de la organización en valores, posibilita la alternativa estratégica, determina las barreras y el factor crítico de éxito de la organización, qué se corresponde con el valor más representativo y que adquirió la condición de principio de la organización. Al finalizar esta primera etapa se confecciona la misión de la entidad sustentada en valores.

La segunda etapa se corresponde con el futuro, donde es preciso determinar los valores deseados, se aplica la técnica de tormentas de ideas (Brainstorming), ${ }^{11}$ luego del análisis de referentes oficiales tales como: el Concepto Revolución, el de Colectivo Moral y el Programa Director para el reforzamiento de valores fundamentales en la sociedad cubana actual, en los que se orientan los valores a los que se debe aspirar. De esta forma se expresan, en el marco de la institución, los valores deseados por el país y la salud pública en particular. Este sistema de valores deseados permite guiar a la organización hacia un deber ser ético, deseado por toda la sociedad. Por otra parte se determinan también valores deseados a partir de los antivalores que se obtienen en el presente.

En esta etapa se construyen los escenarios futuros a través de hipótesis que se someten a un cálculo probabilístico para conformar escenarios a partir de hipótesis de techo, de piso y de incertidumbres. Teniendo en cuenta los valores deseados internamente y el entorno, expresado en los escenarios, se elabora la visión basada en valores, que asocia, los valores deseados con los escenarios futuros.

Para finalizar, en la tercera etapa de la planeación estratégica, se determinan las áreas de resultados clave que coinciden con los campos de salud enunciados anteriormente. Teniendo en cuenta esta reducción previa, ahora estas áreas se organizan de acuerdo con su índice de motricidad (IM) e índice de dependencia (ID) con ayuda de una matriz de impactos cruzados o multiplicación aplicada a una clasificación (MIC-MAC) [Godett M. Prospectiva y Planificación Estratégica. La Habana, selección de páginas; 1985, fotocopia]. 
Luego de obtener las áreas de resultados clave, se procede a construir los objetivos estratégicos correspondientes a cada área. Estos permiten trazar el camino para el alcance de la visión y el estado deseado.

En la construcción de los objetivos estratégicos, se asocian los valores éticos deseados como determinantes en el alcance de una conducta moral adecuada y necesaria para lograr los fines organizacionales. Al mismo tiempo, los valores éticos actuales, son las vías para el alcance de los valores deseados.

Así, quedan elaborados los valores instrumentales los cuales son una síntesis de los valores actuales y los deseados. Al final, para cada valor instrumental, se argumentan criterios de medidas, como modos de actuación o la definición operacional en correspondencia con los valores deseados.

En general, los valores actuales representan el presente, los valores deseados, el futuro. Los valores instrumentales constituyen la síntesis entre ambos.

Finalmente se puede concluir que la metodología para la realización de planes estratégicos a partir de los valores compartidos por los trabajadores en las instituciones de salud, constituye una herramienta útil y de fácil aplicación para la instrumentación práctica de la dirección por valores, que cuenta con instrumentos validados y se aplican técnicas científicas de recogida de la información para evaluar los valores y antivalores compartidos actuales en las organizaciones, determinar los valores deseados y elaborar los valores instrumentales. Ha sido validada por criterio de expertos y por su empleo en la conformación de 141 planes estratégicos de departamentos, servicios e instituciones de salud de la provincia de Guantánamo, con buenos resultados.

Mediante la aplicación de esta metodología los directivos planifican el cambio organizacional a partir de la intención de cambiar el comportamiento moral de los miembros de la organización, al mismo tiempo que se resuelven los problemas y se alcanzan los objetivos y fines.

El alcance del estado deseado se operacionaliza teniendo en cuenta el trazado del modo de actuación correspondiente a cada valor a través de los criterios de medidas.

El Plan Estratégico se hace pertinente a los problemas de salud de la población al dividir este en segmentos manejables de influencia sobre la salud (estilos de vida, biología humana, ambiente y organización de los servicios), y promueve una acción conjunta y cohesionada hacia la solución de esos problemas a través del trazado de objetivos oportunos y apropiados para el objeto social de las instituciones.

\section{REFERENCI AS BI BLI OGRÁFI CAS}

1. Drucker P. La gerencia de las empresas. E.U.A: Editorial Sudamericana; 1970.

2. Guevara E. El socialismo y el hombre en Cuba. La Habana: Editora Política; 1980.

3. Castro Ruz F. Discurso pronunciado en el acto central por el XXV Aniversario de la Victoria de Girón, efectuado en el Teatro "Karl Marx". Granma. 19 Abril de 1986. 
4. Cuba. Partido Comunista de Cuba. Comité Central. Programa director para el reforzamiento de valores fundamentales en la sociedad cubana actual. La Habana: Editora Política; 2006.

5. Vega OJ. Plan Estratégico de la dirección de salud del municipio Guantánamo, mayo 2006. Guantánamo: Facultad de Ciencias Médicas;2006

6. Pardo Fernández A. Modelo sostenible para la integración de la universidad con los procesos sociales. Santiago de Cuba: Universidad de Oriente;2002. [Centro de Estudio "Manuel F. Gram"].

7. González R. Humanismo, espiritualidad y ética médica. La Habana: Editorial de Ciencias Médicas; 2002.

8. Fabelo JR. Los valores y sus desafíos actuales. La Habana: Editorial de Ciencias Médicas; 2004.

9. Álvarez R. Pautas conceptuales y metodológicas para explicar los determinantes de los niveles de salud en Cuba. Rev Cubana Salud Pública. 2007;33(2).

10. Pardo Fernández A. Matriz de Balance de fuerzas innovada. BAFI [citado 26 Mar 2008]. Disponible en: http://www.sld.cu/galerias/doc/sitios/infodir/la_matriz_bafi_2005.doc

11. Osborrn AF. Apllied Imagination. USA: Ed. Scribner; 1998.

Recibido: 2 de septiembre de 2008 .

Aprobado: 23 de octubre de 2008.

Yuslay Ponce Sánchez. Centro Provincial de la Escuela Nacional de Salud Pública. Guantánamo, Cuba.

E-mail: yuslay@infomed.sld.cu 